Supporting Information

\title{
Stable Parent Anions of Dopamine and Adrenaline: A New Form of Neurotransmitters
}

Chu Gong ${ }^{\dagger}$, Wei Wang ${ }^{\dagger}$, Kit Bowen $*,+$ Xinxing Zhang*,†

$\dagger$ Key Laboratory of Advanced Energy Materials Chemistry (Ministry of Education), Renewable Energy Conversion and Storage Center (ReCAST), College of Chemistry, Nankai University, Tianjin 300071, China.

‡ Department of Chemistry, Johns Hopkins University, Baltimore, MD, 21218, USA.

*To whom correspondence should be addressed

E-mail: kbowen@jhu.edu, zhangxx@nankai.edu.cn 


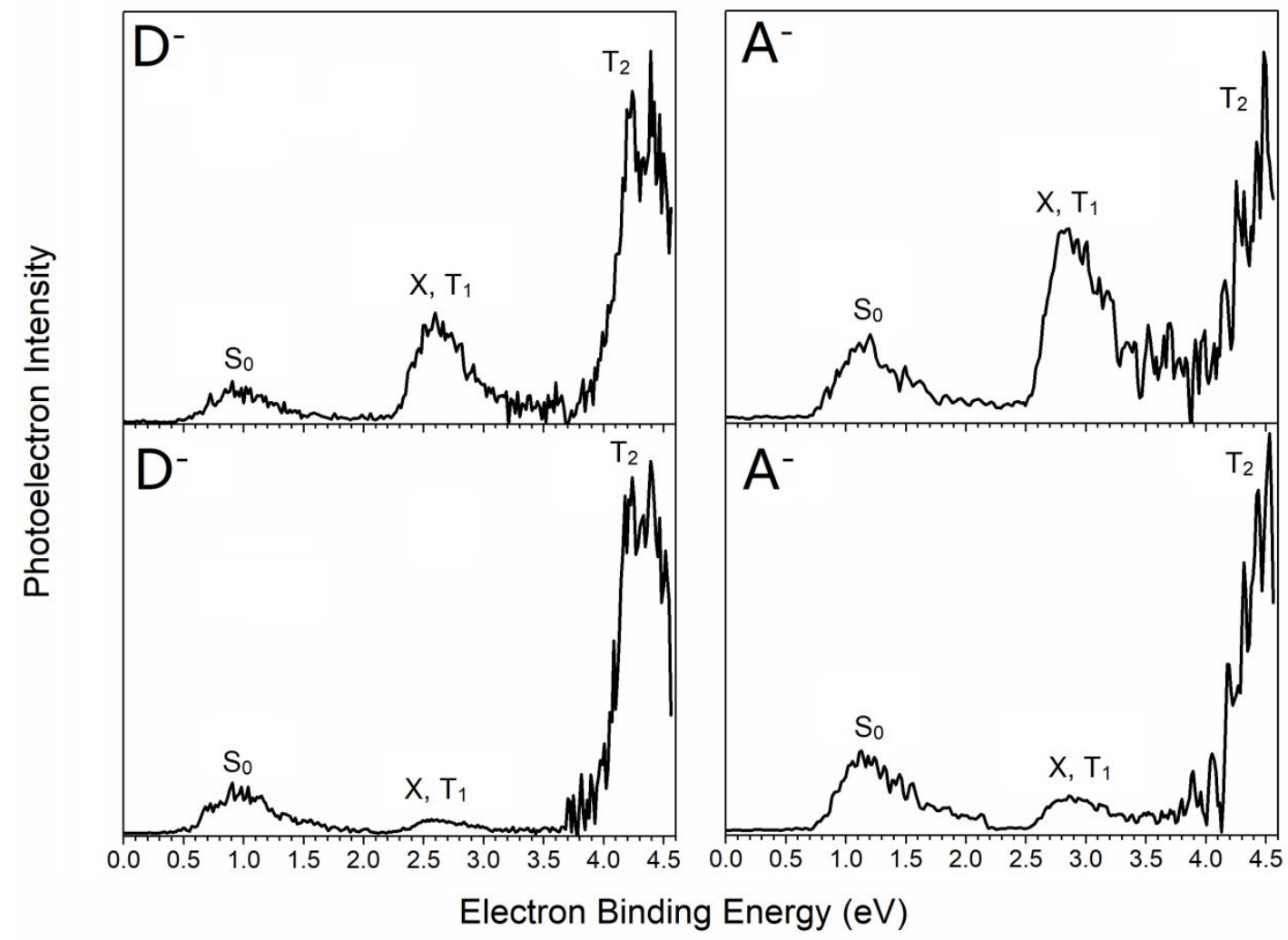

Figure S1. The photoelectron spectra of dopamine anion (D-) and adrenaline anion $\left(\mathrm{A}^{-}\right)$taken on different days' experiments. The change in intensity of peak $\mathrm{X}, \mathrm{T}_{1}$ indicates that it has ${ }^{13} \mathrm{C}$ contamination from the deprotonated molecules $\mathrm{DD}^{-}$and $\mathrm{DA}^{-}$.

\section{Experimental and Theoretical Methods}

Anion photoelectron spectroscopy is conducted by crossing a mass-selected beam of negative ions with a fixed-frequency photon beam and energy-analyzing the resultant photodetached electrons. It is governed by the energy-conserving relationship, hv $=\mathrm{EBE}+\mathrm{EKE}$, where $\mathrm{h} v$ is the photon energy, EBE is the electron binding (transition) energy, and EKE is the electron kinetic energy. Our anion photoelectron spectrometer, which has been described previously, ${ }_{1}^{1}$ consists of a laser vaporization anion source, a linear time-of-flight mass analyzer/selector, a pulsed $\mathrm{Nd}$ :YAG photodetachment laser, and a magnetic bottle electron energy analyzer. Photoelectron spectra were calibrated against the well-known spectrum of $\mathrm{Cu}^{-} .^{2}$ Parent anions of dopamine and adrenaline were generated in a laser vaporization source. Briefly, a silver rod was coated by a layer of dopamine- $\mathrm{HCl}\left(99 \%\right.$, Alfa Aesar $\left.^{\circledR}\right)$ or adrenaline $(98 \%$, Alfa Aesar $^{\circledR}$ ) powder, and then ablated by a pulsed Nd:YAG laser beam operating at a wavelength of $532 \mathrm{~nm}$. We speculate that the role of the silver rod was to supply photoemitted electrons. This laser vaporization method has been proven to be an efficient means to generate organic molecules into the gas phase. ${ }^{3-6}$ The resulting plasma was cooled by supersonically expanding a plume of helium gas from a pulsed gas valve (backing pressure of $60 \mathrm{psi}$ ). Negatively charged anions were then extracted into the spectrometer prior to mass selection and photodetachment.

Density functional theory calculations were conducted by applying Becke's three-parameter hybrid functional (B3LYP) ${ }^{7-9}$ using the Gaussian09 software package ${ }^{10}$ to determine the geometries of both neutral and anionic dopamine and adrenaline as well as the vertical detachment energy (VDE) values. The energies for the vertical transitions from the anions to the excited triplet states of the neutral molecules were calculated with the $\triangle$ SCF-TDDFT method. ${ }^{11}$ All geometries, including those of the anions and neutral molecules, were fully optimized without any geometrical constraints using the 6-31+G $(d, p)$ basis set ${ }^{12}$ and then improved by single point energy calculations with a larger basis set, $6-311++\mathrm{G}$ 
(3df, 3pd). ${ }^{13}$ Since our computational goal here is only to find the electronic structure information (which is also the information obtained from experiment), and the largest energy difference in different conformers is only reported to be within $0.1 \mathrm{eV},{ }^{14,15}$ which is not at the same order of magnitude of the electronic energy levels (several $\mathrm{eV}$ ), therefore, for structural optimizations, the starting conformations of all the neutral and anionic isomers in this study were simply obtained from published studies using the most stable conformations. ${ }^{14,15}$ The energies of the anions having similar structures as the neutral (i.e. without tautomerization) were also calculated to confirm that they cannot exist due to very high energy. Since both dopamine and adrenaline have two acidic hydroxyl groups on the benzene ring, one of them, or both of them could migrate to different carbon atoms on the benzene ring, hence, an extended library of tautomers were generated by a combinatorial structural search. The right tautomers were those where one $\mathrm{H}$ atom migrates to adjacent $\mathrm{C}$ atom, which is validated by calculating the VDE values and comparing with experimental results. The VDE is calculated to be the energy difference between the ground state of the anion and the neutral with the same structure as the anion.

\section{Table S1. 3D coordinates of all the species calculated.}

D:

C $2.08642200-0.288967000 .09270400$

C $1.55302900-1.573621000 .11789200$

C $0.17747300-1.76490100-0.05252100$

C $-0.69070500-0.68415100-0.25435500$

C $-0.141759000 .61028400-0.28043500$

C $1.224226000 .80080000-0.10486000$

H $2.22230600-2.413733000 .27374100$

H $-0.22020400-2.77601400-0.02811000$

H $-0.802184001 .46330000-0.41350600$

C $-2.17692300-0.91756900-0.44791900$

H $-2.37554100-1.98999400-0.33816100$

$\mathrm{H}-2.47006900-0.66211500-1.47767500$

C $-3.10827900-0.158414000 .51620700$

H -2.69985200 -0.21206100 1.53149800

O $1.836461002 .04021400-0.11226000$

O $3.43117000-0.098526000 .26003200$

H $1.177524002 .73565300-0.23243600$

H 3.613089000 .852177000 .21599200

$\mathrm{N}-3.223452001 .264402000 .15271400$

$\mathrm{H}-3.756985001 .36810100-0.70791600$

$\mathrm{H}-3.721736001 .781452000 .87233200$

H $-4.08412200-0.671856000 .53278000$

D- iso 1:

C $2.097683000 .34302200-0.15432600$

C $1.469962001 .59049100-0.20370800$

C 0.069879001 .664185000 .04602400

C -0.710793000 .561768000 .31920300$

C $1.41524500-0.860511000 .13869000$

H $2.046299002 .48438400-0.42496100$

$\mathrm{H}-0.403521002 .647885000 .02530700$

C -2.167313000 .710242000 .66143400$

$\mathrm{H}-2.380032001 .769442000 .87198900$

$\mathrm{H}-2.398611000 .163683001 .60036100$

C $-3.213970000 .26175900-0.38177300$

H $-2.982462000 .73401400-1.34446600$

O $2.05997100-1.971045000 .18059700$

O $3.456502000 .20482400-0.38754500$

H $3.57418200-0.76385900-0.27265100$

N $-3.21901000-1.19472500-0.58468400$

$\mathrm{H}-3.30342400-1.677586000 .30736800$

H -3.99992500 - $1.48348100-1.16738100$ 
$\mathrm{H}-4.201899000 .64684300-0.05405300$ C $-0.07971200-0.813604000 .41489200$ $\mathrm{H}-0.57730600-1.52634900-0.26296000$ H $-0.25790000-1.246243001 .42720700$

$\mathrm{D}^{-}$iso 2 :

C $2.16555100-0.212157000 .12368600$ C $0.12080200-1.71289800-0.08305000$ C $-0.66229200-0.60416800-0.31585400$ C $1.277753000 .85909500-0.12229500$ H $-0.33607100-2.70351600-0.08412300$ C - $2.14998000-0.79130400-0.55319500$ H $-2.35352900-1.86742700-0.62724400$ H $-2.44162900-0.35540400-1.52361600$ C $-3.08808700-0.203763000 .52362100$ $\mathrm{H}-2.58951700-0.255066001 .49785100$ O $1.871656002 .11108300-0.14051700$ O 3.420114000 .012991000 .30852300 $\mathrm{N}-3.412164001 .209277000 .26129900$ H -3.95355200 $1.29715800-0.59575700$ $\mathrm{H}-3.965653001 .605861001 .01613500$ $\mathrm{H}-3.99656400-0.833865000 .58726800$ C $1.60744500-1.626846000 .17156700$ H $1.87667700-2.065742001 .15440200$ H $2.17810900-2.24313000-0.55224900$ C $-0.096559000 .70936600-0.34144300$ H $-0.725710001 .57825600-0.50010400$ H 2.810627001 .881382000 .03608100
A:
C $2.72900900-0.614624000 .05617100$
C $0.50078100-1.43110400-0.40422700$
C $-0.01349900-0.13009200-0.35322600$
C 2.218317000 .691715000 .09573500
$\mathrm{H}-0.16040600-2.26738400-0.61671600$
C $-1.498743000 .11627700-0.51904600$
O 3.152597001 .680273000 .33700700
C $0.862777000 .93391400-0.10197700$
H $0.473463001 .94815000-0.08517500$
C $1.86112900-1.67462800-0.19577500$
H $2.26598000-2.68063800-0.23790700$
O $4.06181200-0.852516000 .24916200$
C - $2.27035500-0.158620000 .79390000$
$\mathrm{H}-1.803250000 .431305001 .58980300$
H -2.17833700 -1.22394600 1.06278900
$\mathrm{N}-3.663881000 .277723000 .65717500$
H $4.50814300-0.003566000 .38739300$
H 2.738137002 .551186000 .29327200
$\mathrm{H}-1.87454400-0.56790500-1.29778200$
O $-1.738926001 .46037500-0.91518400$
H -2.65600000 $1.64004500-0.63652800$
$\mathrm{H}-4.031618000 .564013001 .55800500$
C $-4.56045700-0.698656000 .03549500$
H $-5.58015400-0.303436000 .03025800$
$\mathrm{H}-4.26765000-0.86624600-1.00553000$
H -4.56420600 -1.67578100 0.54771300
$\mathrm{A}^{-}$iso 1 :
C $2.77106600-0.58544500-0.06981900$
C $0.46719600-1.284817000 .25845200$
C -0.013720000 .003963000 .14911800$ 
C $2.408788000 .77611000-0.19157500$

$\mathrm{H}-0.22603400-2.110181000 .41212200$

C -1.464608000 .361778000 .19453500$

O $3.308643001 .66273200-0.41712700$

C $1.84726300-1.609673000 .16077400$

H $2.18460300-2.638549000 .24719400$

O $4.12271900-0.84862200-0.20736800$

C $-2.44525200-0.805619000 .01013200$

H $-2.29339300-1.521354000 .82403600$

H $-2.23869600-1.31895200-0.94374000$

$\mathrm{N}-3.84383900-0.338175000 .09083400$

H $4.482633000 .05316500-0.35696300$

H - $1.656619001 .09323900-0.61638200$

O -1.799913001 .036463001 .44260900$

H -2.77153900 1.083094001 .44668300

H $-4.43742800-1.094953000 .41531100$

C $-4.377188000 .19630300-1.15999800$

H $-5.430669000 .46527300-1.02465600$

$\mathrm{H}-3.834955001 .10583100-1.43622100$

H $-4.30062400-0.50713400-2.01006300$

C $0.947461001 .16459400-0.02811300$

H 0.876433001 .867523000 .82342700

H $0.655547001 .78248300-0.90254300$

$\mathrm{A}^{-}$iso 2:

C $2.83055900-0.447622000 .16151300$

C $0.57746700-1.52280100-0.32630000$

C $0.00138900-0.27258500-0.36152800$

C 2.144486000 .786737000 .09670800

H $-0.03985000-2.40369600-0.50514400$

C - $1.49593700-0.16435600-0.54743500$

O 2.938574001 .904440000 .29398900

O $4.09516700-0.474306000 .39468500$

C -2.190512000 .349890000 .72896000$

$\mathrm{H}-1.746916001 .314454000 .99288800$

$\mathrm{H}-1.99111000-0.353238001 .55464600$

$\mathrm{N}-3.635148000 .559557000 .50755600$

$\mathrm{H}-1.87852000-1.17360800-0.77312000$

O $-1.843751000 .71148500-1.63436600$

H $-2.753111000 .99813400-1.44154700$

$\mathrm{H}-3.981877001 .262120001 .15283900$

C $-4.44710800-0.650525000 .61366100$

$\mathrm{H}-5.50581600-0.396336000 .49270100$

H $-4.17997600-1.34748300-0.18671800$

$\mathrm{H}-4.32111900-1.183874001 .57393700$

C $0.773367000 .91034800-0.14786600$

H $0.321430001 .89371800-0.22906800$

C $2.04686100-1.73476300-0.05277600$

H $2.20551300-2.382492000 .83403800$

H $2.53356600-2.29755200-0.87466800$

H 3.820467001 .494041000 .43223000

\section{References}

(1) Zhang, X.; Wang, Y.; Wang, H.; Lim, A.; Ganteför, G.; Bowen, K. H.; Reveles, J. U.; Khanna, S. N. On the existence of designer magnetic superatoms. J. Am. Chem. Soc. 2013, 135, 4856-4861.

(2) Ho, J.; Ervin, K. M.; Lineberger, W. C. Photoelectron spectroscopy of metal cluster anions: $\mathrm{Cu}^{-}$, $\mathrm{Ag}^{-}$, and $\mathrm{Au}^{-}$.J. Chem. Phys. 1990, 93, 6987-7002. 
(3) Li, X.; Bowen, K. H.; Haranczyk, M.; Bachorz, R. A.; Mazurkiewicz, K.; Rak, J.; Gutowski, M. Photoelectron spectroscopy of adiabatically bound valence anions of rare tautomers of the nucleic acid bases. J. Chem. Phys., 2007, 127, 174309/1-174309/6.

(4) Zhang, X.; Tang, X.; Bowen, K. H. Photoelectron spectroscopic study of the ethyl cyanoacrylate anion. Chem. Phys. Lett. 2013, 582, 21-23.

(5) Zhang, X.; Tang, X.; Mayo, D. H.; DeCarlo, S.; Eichhorn, B.; Bowen, K. H. Photoelectron spectroscopic study of the diphenylphosphide anion and its oxide. Chem. Phys. Lett. 2014, 597, $110-113$

(6) Popov, I. A.; Zhang, X.; Eichhorn, B. W.; Boldyrev, A.; Bowen, K. H. Aluminum chain in $\mathrm{Li}_{2} \mathrm{Al}_{3} \mathrm{H}_{8}{ }^{-}$as suggested by photoelectron spectroscopy and $a b$ initio calculations. Phys. Chem. Chem. Phys. 2015, 17, 26079-26083.

(7) Becke, A. D. Density-functional exchange-energy approximation with correct asymptotic behavior. Phys. Rev. A. 1988, 38, 3098-3100.

(8) Becke, A. D. Density-functional thermochemistry. III. The role of exact exchange. J. Chem. Phys. 1993, $98,5648-5652$.

(9) Lee, C.; Yang, W.; Parr, R. G. Development of the Colic-Salvetti correlation-energy formula into a functional of the electron density. Phys. Rev. B. 1988, 37, 785-789.

(10) Risch, M. J.; Trucks, G. W.; Schlegel, H. B.; Scuseria, G. E.; Robb, M. A.; Cheeseman, J. R.; Scalmani, G.; Barone, V.; Mennucci, B.; Petersson, G. A. et al. Gaussian 09, revision A.1; Gaussian, Inc.: Wallingford, CT, 2009

(11) T. Ziegler, T.; Rauk, A.; Baerends, E. J. On the calculation of multiplet energies by the HartreeFock-Slater method. Theor. Chim. Acta 1977, 43, 261.

(12) Hehre, W. J.; Ditchfield, R.; Pople, J. A. Self-consistent molecular orbital methods. XII. Further extensions of Gaussian-type basis sets for use in molecular orbital studies of organic molecules. $J$. Chem. Phys. 1972, 56, 2257-2261.

(13) Krishnan, R.; Binkley, J. S.; Seeger, R.; Pople, J. A. Self-consistent molecular orbital methods. XX. A basis set for correlated wave functions. J. Chem. Phys.1980, 72, 650-654.

(14) Cabezas, C.; Peña, I.; López, J. C.; Alonso, J. L. Seven conformers of neutral dopamine revealed in the gas phase. J. Phys. Chem. Lett. 2013, 4, 486-490.

(15) Mourik, T. V. The shape of neurotransmitters in the gas phase: A theoretical study of adrenaline, pseudoadrenaline, and hydrated adrenaline. Phys. Chem. Chem. Phys. 2004, 6, 2827-2837. 